(4) Hotz, W.; Strobl, G. Colloid Polym. Sci., in press.

(5) Ferry, J. D. Viscoelastic Properties of Polymers; Wiley: New York, 1980.

(6) Benthack-Thoms, H. Dissertation; Clausthal-Zellerfeld, 1985.

(7) Heppke, G.; Schneider, F. Z. Naturforsch. 1972, 27a, 976.

(8) Kresse, H.; Talrose, R. Makromol. Chem., Rapid. Commun. $1981,2,369$.

(9) Zentel, R.; Strobl, G.; Ringsdorf, H. Macromolecules 1985, 18, 960.

(10) Haase, W.; Pranoto, H.; Bormuth, F. Ber. Bunsenges. Phys. Chem. 1985, 89, 1229.

(11) Attard, G. S. Mol. Phys. 1986, 58, 1087.

(12) Martin, A. J.; Meier, G.; Saupe, A. Symp. Faraday Soc. 1971, 5,119 .
(13) Zeller, H. Phys. Rev. Lett. 1982, 48, 334.

(14) Attard, G.; Moura-Romas, J.; Williams, G. J. Polym. Sci., Polym. Phys. Ed. 1987, 25, 1099.

(15) Doi, M.; Edwards, S. F. The Theory of Polymer Dynamics; Clarendon: Oxford, 1986.

(16) Hess, S. Z. Naturforsch. A 1975, 30, 1224.

(17) Kneppe, H.; Schneider, F.; Sharma, N. K. J. Chem. Phys. $1982,77,3203$

(18) Zeller, H. R. Phys. Rev. A 1981, 23, 1434

(19) Kresse, H.; Stettin, H.; Kostromin, S.; Shibaev, V. Mol. Cryst. Liq. Cryst., submitted for publication.

Registry No. $\mathrm{C}_{6} \mathrm{H}_{13} \mathrm{OC}_{6} \mathrm{H}_{4}-p-\mathrm{CO}_{2} \mathrm{C}_{6} \mathrm{H}_{4}-p-\mathrm{OC}_{3} \mathrm{H}_{7}, 50649-51$ -

\title{
Density Profile of Terminally Anchored Polymer Chains: A Monte Carlo Study
}

\author{
Amitabha Chakrabarti ${ }^{*}$ and Raul Toral ${ }^{\dagger}$ \\ Department of Physics and Center for Polymer Science and Engineering, Lehigh University, \\ Bethlehem, Pennsylvania 18015. Received July 13, 1989; \\ Revised Manuscript Received September 29, 1989
}

\begin{abstract}
We present results of a detailed Monte Carlo simulation study of a system of a large number of polymer chains terminally anchored or end-grafted on a flat surface. We study this system on a three-dimensional lattice for several different values of the surface coverage and the chain length. We also consider several different distributions for the chain lengths. For monodisperse chains, we find that the monomer density profile shows a depletion layer near the grafting plane in agreement with phenomenological theories. Beyond this depletion layer, the density profile can be represented by a parabolic form. This result is in agreement with recent self-consistent-field (SCF) calculations rather than with the scaling arguments that predict a plateau region for the density profile. The chain-end density is also found to be consistent with the SCF calculations; i.e., we find that the free ends of the chains are not excluded from regions near the grafting surface. We also study the effect of polydispersity in the chain lengths. In the case of a system consisting of two species of polymers of length $N$ and $2 N$, we find that there is a region in which the density profile matches that of the monodisperse case with chain length $N$, in agreement with another recent self-consistent-field calculation. The width of this region, however, is narrower than that predicted by the theory. We have also considered a uniform distribution of chain lengths and compared the density profile with the functional form obtained by intergrating the equations derived in the SCF formalism. The agreement between the Monte Carlo data and the theory is remarkable except, again, for the presence of a depletion layer near the grafting plane.
\end{abstract}

\section{Introduction}

The configurations of polymer chains adsorbed at a solid-liquid or liquid-liquid interface have attracted considerable interest in recent years, mainly because of their important applications in various different problems of colloidal stability, adhesion, lubrication, and biophysics. Colloidal particles are kept in suspension and often protected against flocculation by terminally anchoring (also called grafting) polymers onto their surfaces. ${ }^{1-5}$ Since the adsorbed polymer layers repel each other, they can provide a long-range repulsion between the colloidal particles. A quantitative prediction about the interactions between the stabilized particles for different solvent qualities and polymer-surface interactions is an extremely difficult task, since this will involve a detailed knowl-

\footnotetext{
* Present address: Department of Physics, Kansas State University, Manhattan, KS 66506.

${ }^{+}$Permanent address: Departament de Fisica, Universitat de les
} Illes Balears, Palma de Mallorca, E-07071 Spain. edge of the chain conformations near the surface and a quantitative theory of polymer solution dynamics, none of which is presently attainable. As a result, previous theoretical treatments of surfaces bearing terminally attached polymer chains have employed various approximate schemes, such as Flory-type mean-field arguments, ${ }^{6}$ phenomenological scaling arguments, ${ }^{7}$ and selfconsistent-field (SCF) methods. ${ }^{8-10}$ Among these various approaches, the SCF methods seem to be in better agreement with the experimental observations. ${ }^{11}$

Both Flory-type arguments of Alexander ${ }^{6}$ and scaling arguments of de Gennes ${ }^{7}$ note that, under good solvent conditions and high enough surface coverage $\sigma$, allowing overlap between individual chains, the chains will be stretched, for the case of nonadsorbing grafting surfaces. These theories do not provide an explicit form for the equilibrium monomer density profile as a function of distance $z$ from the grafting plane (measured in units of monomer size a). The qualitative features are 
the following: a depletion zone of width $\xi \sim \sigma^{-1 / 2}$ near the surface beyond which the density increases as $\sigma z^{2 / 3}$ until it reaches a plateau of value proportional to $\sigma^{2 / 3}$. For chains with molecular weight $N$, this plateau extends up to a distance $h^{*} \sim N \sigma^{1 / 3}$ where the density decreases to zero. In the SCF theory of Dolan and Edwards, ${ }^{8}$ a diffusion-type equation is solved to obtain the configurations of terminally attached chains. Although some limiting cases of the Dolan-Edwards SCF method can be handled analytically, one needs to solve the corresponding equations numerically in the more general cases. Recent numerical study ${ }^{13}$ of this theory indicates that for nonadsorbing surfaces and good solvent conditions, the monomer density profile is substantially different from the form predicted by Alexander and de Gennes. Actually, the authors in ref 13 find that the density profile shows a maximum near the grafting surface and then monotonically decreases to zero instead of showing a plateau region. In the SCF theories of Scheutjens and Fleer, ${ }^{9}$ polymer configurations are modeled as stepweighted random walks on a lattice and the concentration profile is calculated by an iterative matrix procedure. Numerical calculations, ${ }^{14,15}$ based on the Scheutjens and Fleer SCF method, have also been carried out. These studies also indicate that for nonadsorbing surfaces and good solvents the monomer density profile deviates from the one predicted by Alexander-de Gennes, showing, again, a maximum near the grafting surface. Recently, Milner, Witten, and Cates $^{16}$ (hereafter to be referred as MWC) have simplified the SCF formalism on the assumption of strongly stretched chains and weak excluded-volume interactions and found a parabolic form for the concentration profile by analytical calculations. The authors have compared their results with those obtained from numerical solutions of Scheutjens-Fleer SCF-type equations ${ }^{14}$ and claimed good agreements, although we note that the MWC calculation does not reproduce the maximum in the density profile for distances close to the grafting surface, observed by the methods described before. Since all the SCF-type calculations use essentially a meanfield approach, we believe that independent numerical computations of the density profile will be quite useful to understand the validity of the SCF-type calculations or, for that purpose, Flory- and scaling-type calculations as well.

With an eye to this direction, Monte Carlo ${ }^{15,17}$ and molecular dynamics ${ }^{18}$ simulations have been carried out. The molecular dynamics calculations seem to support the parabolic density profile for not too large surface coverages and for distances not too close to the grafting surface, in which regime the calculations are not very precise. Also, in the molecular dynamics simulation ${ }^{18}$ one needs to consider a short-range repulsive interaction between the grafting surface and the monomers whereas in the MWC theory no such interactions are assumed. Moreover, ref 18 considers monodisperse polymer chains only, and no detailed comparison with theory ${ }^{19}$ was carried out for other distributions of chain lengths. In previous Monte Carlo simulations one finds a density profile showing a maximum near the grafting surface. However, these Monte Carlo simulations are somewhat inconclusive, since the authors have studied single chain configurations and used the periodic boundary conditions to mimic the effect of surface coverages, and it is not clear at all whether these studies provide quantitative informations about the density profile when the actual surface coverage is varied.

In this paper, we report results of a detailed Monte Carlo simulation study of a system of a large number of polymer chains end-grafted onto a surface, for several values of the surface coverage and chain length. We consider different probability distribution functions for the chain lengths: a monodisperse distribution, a bimodal distribution, and a uniform distribution between a minimum and a maximum value for the chain length $N$. For monodisperse chains, we find that the monomer density profile can be represented by a parabolic form, except for a depletion zone very close to the grafting surface. The width of this depletion zone is consistent with that predicted by scaling theories. ${ }^{7}$ We also compute the density of the free chain ends as a function of distances from the grafting surface and find that there is no "dead zone"; i.e., the free ends are not excluded from regions near the grafting surface, in agreement with SCF calculations of MWC and the molecular dynamics calculations. ${ }^{18} \mathrm{We}$ also compare the results for the density profile in the case of bimodal and uniform distributions with those of another recent SCF-type calculations. ${ }^{19}$ We find good agreement with the theory except, again, for the presence of a depletion zone of width of a few monomer spacings near the surface.

The rest of the paper is organized as follows: in section II we describe the model and the numerical methods used in this study, in section III we present the results for both monodisperse and polydisperse chains, and finally, in section IV, we conclude with a brief summary and conclusions.

\section{The Model and Numerical Procedure}

In order to simulate the behavior of many polymer chains terminally anchored to a surface, we have carried out extensive Monte Carlo calculations. We choose a simple cubic lattice consisting of $L_{x} \times L_{y} \times L_{z}$ sites, with $L_{x}=L_{y}=$ 80 and $L_{z}=100$ and use periodic boundary conditions in the $x$ and $y$ directions. The grafting (and otherwise nonadsorbing) surface is located at $z=0$, and a second, also nonadsorbing, surface is situated at $z=99$. During the simulations, the chains are not allowed to cross these surfaces; otherwise, there is no explicit interactions between the chains and the surfaces. The second surface is placed at a far enough distance from the grafting plane such that it does not affect the chain configurations for the chain lengths considered in the simulations. Thus, effectively, our results correspond to the limit $L_{z} \rightarrow \infty$. We study systems of polymer chains consisting of $N+1$ monomers with $N=49$ and 99 for the monodisperse case, for several values of surface coverage $\sigma$. The number of polymer chains present in the system is then given by $N_{t}=$ $\sigma A$, where $A=L_{x} L_{y}$ is the area of the grafting plane. The maximum value of $\sigma$ considered in the simulations is 0.12 , for which case there are 768 chains in the system. All the chains in the simulations obey excluded-volume interactions, such that a particular lattice site cannot be occupied by more that one chain at a time. We have chosen random intial conditions for the simulation; i.e., for a particular run, one end of each of the $N_{t}$ chains is kept fixed at a randomly chosen vacant site on the grafting surface. The results obtained are then averaged over 10 such initial configurations in each case.

In the presence of excluded-volume interactions and for large values of $\sigma$ and $N$, it is extremely time-consuming to randomly end-graft a large number of chains. We follow a modified version of the existing method ${ }^{20}$ of growing and equilibrating chains simultaneously. At each step we try to put a complete chain as a self-avoiding random walk starting from a randomly chosen vacant site on the grafting surface. If the trial fails, all the chains already present in the system are equilibrated by one unit of time. 
We define our unit of time, in Monte Carlo steps per monomer (MCM), as $N_{t}$ " $N$-bead cycles", i.e. $N_{t} N$ elementary bead jump attempts. The particulars of an elementary bead jump attempt depend on the local environment of the randomly chosen monomer and could be one of the following: ${ }^{21,22}$ an end bead jump, a normal internal bead jump, or a crankshaft motion. After all the required chains are grafted, the final equilibrating process starts. The starting configurations, at this stage, are equilibrated over times much large than the longest relaxation time of an individual chain with excluded-volume interactions. It is well-known ${ }^{22}$ that for a chain with $N$ +1 beads and under excluded-volume interactions, the largest relaxation time $\tau_{\text {single }}$ is given by, in units of $N$ bead cycles,

$$
\tau_{\text {single }} \approx 0.25 N^{2.13}
$$

In the monodisperse case, we have equilibrated the system for a time $\tau=10 \tau_{\text {single }}$ MCM. Which means that, on average, every chain has been equilibrated 10 times the largest relaxation time for a single chain. When, for example, $N=99$ and $\sigma=0.12$ (correspondingly, $N_{t}=$ 768 ), the system has been equilibrated for about 45000 MCM, or 3.4 billion monomer updates. This is a much more extensive calculation than any of the previous Monte Carlo studies of polymer systems known to us. For the polydisperse case, the equilibration time $\tau$ is chosen to be 10 times $\tau_{\max } \mathrm{MCM}$, where $\tau_{\max }$ is the value of $\tau_{\text {single }}$ for the maximum $N$ in the sample. After the equilibration is done, several different quantities such as monomer density and free chain-end density as a function of distances from the grafting plane are computed over another time $\tau_{\mathrm{m}}=10000 \mathrm{MCM}$. These quantities are then averaged over 10 different random initial conformations of the chains.

\section{Results}

A. Monodisperse Case. The monomer density $\varphi(z)$ as a function of distance $z$ from the grafting plane, for several values of the surface coverage $\sigma$, is shown in parts a and b of Figure 1, for chain lengths $N=49$ and $N=$ 99 , respectively. For all values of $\sigma$ considered here the density profile shows a maximum close to the grafting plane, also seen in previous Monte $\mathrm{Carlo}^{15,17}$ and numerical SCF ${ }^{13-15}$ calculations. Although we cannot precisely determine how the location $\xi$ of the maximum varies with coverage, we observe that the values of $\xi$ (a few lattice spacings) are in rough agreement with the relation $^{7} \xi=\sigma^{-1 / 2}$. We also note that beyond these maxima the $\varphi(z)$ curves can be approximated by a parabolic form $\varphi(z)=A_{0}-B_{0} z^{2}$ for all values of $\sigma$ and $N$. The quality of the parabolic fit improves as the chain length is increased. Further details of the parabolic fit are listed in Table I. The self-consistent calculations of $\mathrm{MWC}^{16}$ predict a parabolic form for all $z$ up to a maximum value $h$ as

$$
\varphi(z)=\frac{1}{\omega}\left(A-B z^{2}\right) \theta(h-z)
$$

where

$$
\begin{gathered}
A=\frac{N \sigma \omega}{h}+\frac{B h^{2}}{3} \\
B=\frac{\pi^{2}}{8 N^{2}}
\end{gathered}
$$

$\theta(x)$ is a step function of $x$, and, for a system under no
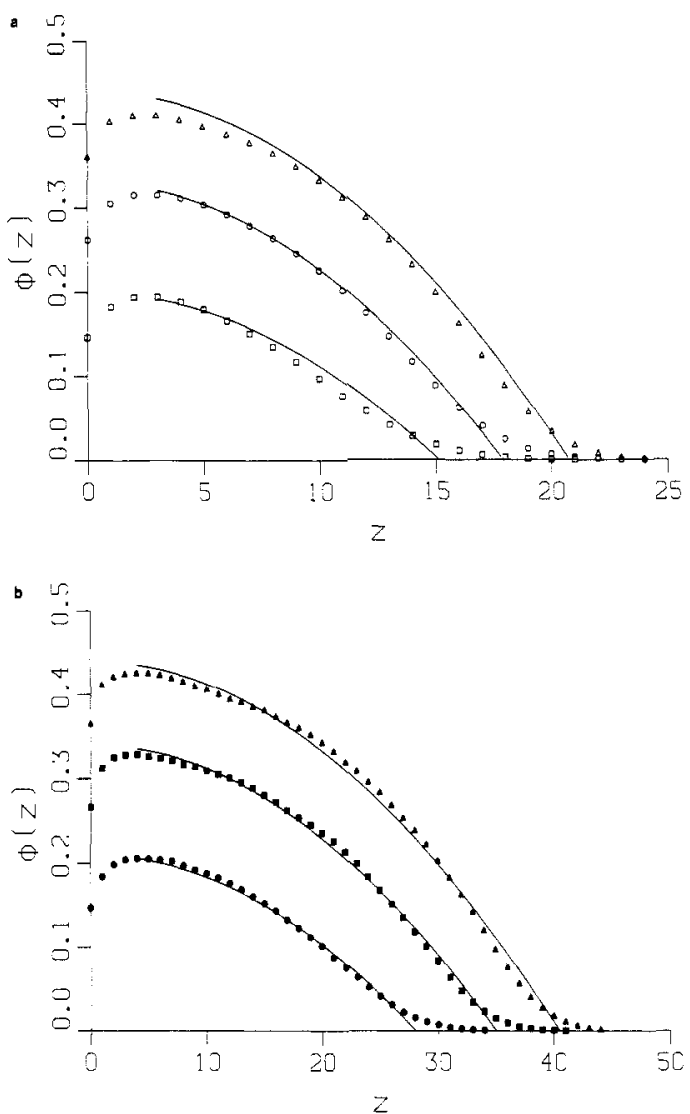

Figure 1. (a) The monomer density profile $\varphi(z)$ vs distance from the grafting plane $z$ for several values of the surface coverage $\sigma$ and for chain length $N=49$. The symbols $\square, 0$, and $\Delta$ correspond respectively to $\sigma=0.04,0.08$, and 0.12 . The solid lines are the parabolic fit to the form $\varphi(z)=A_{0}-B_{0} z^{2}$. The details of the fit are listed in Table I. (b) The monomer den. sity profile $\varphi(z)$ vs distance from the grafting plane $z$ for several values of the surface coverage $\sigma$ and for chain length $N=$ 99. The symbols $\square, 0$, and $\Delta$ correspond respectively to $\sigma=$ $0.04,0.08$, and 0.12 . The solid lines are the parabolic fit to the form $\varphi(z)=A_{0}-B_{0} z^{2}$. The details of the fit are listed in Table I.

external force like the ones considered here

$$
h=\left(\frac{12 \sigma \omega}{\pi^{2}}\right)^{1 / 3} N
$$

Here $\omega$ is the strength of the excluded-volume interaction. The parameters of the parabolic fit are thus determined by this theory as

$$
\begin{gathered}
A_{0}=\left(\frac{9 \pi^{2} \sigma^{2}}{32 \omega}\right)^{1 / 3} \\
B_{0}=\frac{\pi^{2}}{8 N^{2} \omega}
\end{gathered}
$$

These equations lead to the following identities:

$$
\begin{gathered}
\frac{A_{0}{ }^{3}}{B_{0}} \frac{4}{9 \sigma^{2} N^{2}}=1 \\
\omega=\frac{9 \pi^{2} \sigma^{2}}{32 A_{0}{ }^{3}} \\
\omega=\frac{\pi^{2}}{8 N^{2} B_{0}}
\end{gathered}
$$

After computing the best parameters of the parabolic fit $A_{0}$ and $B_{0}$ by a least-squares method, we have checked that eq 8 is well satisfied (see Table I). Then, from eq 9 
Table I

Values of the Parabolic Fit $\varphi(z)=A_{0}-B_{0} z^{2}$ for $N=49$ and $N=99$ for Different Values of $\sigma^{*}$

\begin{tabular}{ccccccccc}
\hline$N$ & $\sigma$ & $z_{\min }$ & $\boldsymbol{z}_{\max }$ & $A_{0}$ & $B_{0}$ & $\left(A_{0}{ }^{3} / B_{0}\right)\left(4 / 9 \sigma^{2} N^{2}\right)$ & $9 \pi^{2} \sigma^{2} / 32 A_{0}{ }^{3}$ & $\pi^{2} / 8 N^{2} B_{0}$ \\
\hline 49 & 0.04 & 3 & 15 & 0.20 & $8.75 \times 10^{-4}$ & 1.058 & 0.555 & 0.587 \\
49 & 0.08 & 3 & 18 & 0.33 & $1.03 \times 10^{-3}$ & 1.009 & 0.494 & 0.499 \\
49 & 0.12 & 3 & 20 & 0.44 & $1.02 \times 10^{-3}$ & 1.074 & 0.469 & 0.504 \\
99 & 0.04 & 4 & 29 & 0.21 & $2.67 \times 10^{-4}$ & 0.983 & 0.471 & 0.452 \\
99 & 0.08 & 4 & 33 & 0.34 & $2.77 \times 10^{-4}$ & 1.005 & 0.454 \\
99 & 0.12 & 4 & 39 & 0.44 & $2.68 \times 10^{-4}$ & 1.001 & 0.469 & 0.470
\end{tabular}

a The parabolic fit is tried beyond the maximum of the density profile and seem to work for distances between $z_{\min }$ and $z_{\max }$ whose values are also listed in the table. Column 7 should be 1 if $A_{0}$ and $B_{0}$ were consistent with MWC. Columns 8 and 9 are different values for $\omega$ according to eqs 9 and 10 (see text).

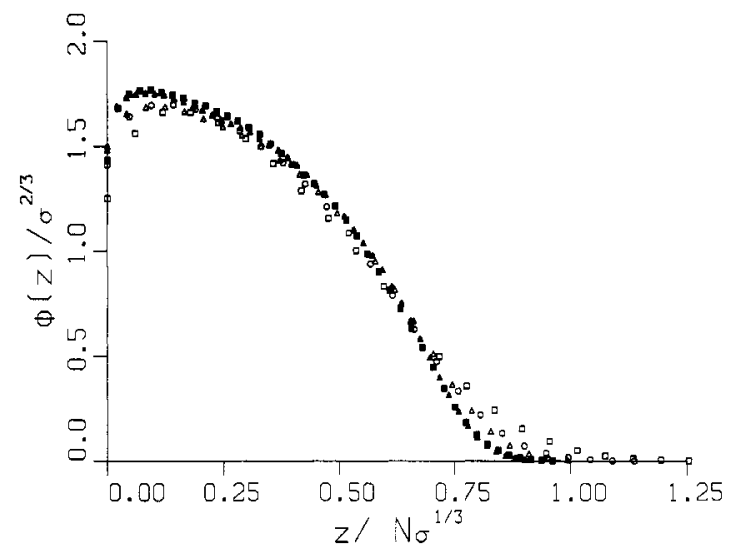

Figure 2. Scaling plot of the density profile for different values of $\sigma$ and $N$. The symbols have the same meaning as in parts $a$ and $b$ of Figure 1 .

and 10 we have determined $\omega$ for any value of $\sigma$ and $N$. The results are also listed in Table I and do not show any systematic dependence on either $\sigma$ or $N$. From this table we extract the value of the excluded-volume-interaction strength for our lattice model as the average of the results listed in columns 8 and 9 of Table I:

$$
\omega=0.50 \pm 0.05
$$

Another equivalent, yet illuminating way of presenting the data is to note that, according to eq 6 and $7, \varphi(0)$ $\sim \sigma^{2 / 3}$ and $h$, the distance at which $\varphi(h)=0$, scales as $N \sigma^{1 / 3}$. It is possible then to rewrite eq 2 in a scaling form

$$
\frac{\varphi(z)}{\sigma^{2 / 3}}=F_{1}\left(\frac{z}{N \sigma^{1 / 3}}\right)
$$

where $F_{1}(x)$ is a scaling function independent of $\sigma$ and $N$. In order to test this scaling prediction, we rescale $\varphi(z)$ by $\sigma^{2 / 3}$ and $z$ by $N \sigma^{1 / 3}$ and plot the corresponding graphs in Figure 2. All the data seem to collapse reasonably well on a single master curve.

Another important claim of MWC is that free ends of the chains are not excluded from regions near the grafting surface. Actually, an analytical form of chain-end density, $\epsilon(z)$, is found in the above calculation: ${ }^{23}$

$$
\epsilon(z)=\frac{\pi^{2}}{4 \omega N^{3}} z\left(h^{2}-z^{2}\right)^{1 / 2} \theta(h-z)
$$

Since we have been able to determine the value of $\omega$ quite accurately from the density profile data, all the parameters in the above equation are known. In order to stress the universal features of this expression, we note that if $\epsilon(z)$ is reduced by $\sigma^{2 / 3} / N$ and $z$ is reduced by $N \sigma^{1 / 3}$, then one should have a scaling curve for the reduced chainend density, which should not depend on either $N$ or $\sigma$.

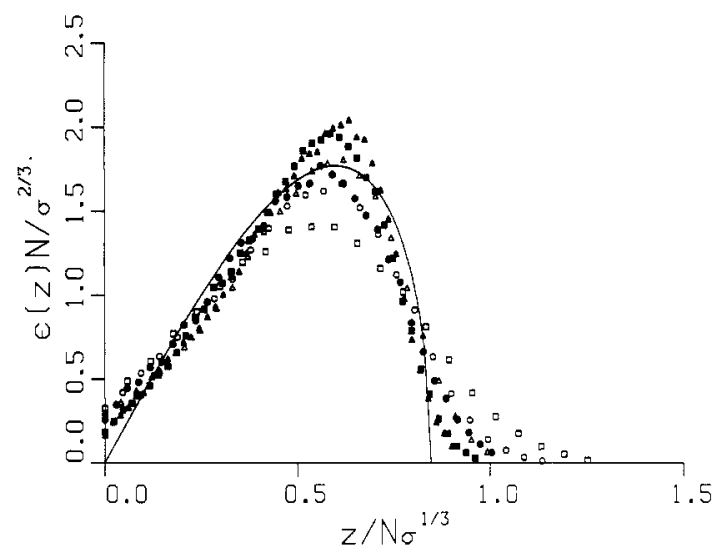

Figure 3. Scaling plot of the density, $\epsilon(z)$, for different values of $\sigma$ and $N$. The solid line is the MWC prediction ${ }^{16}$ eq 15, with $\omega=0.5$. The symbols have the same meaning as in parts $a$ and $\mathrm{b}$ of Figure 1.

Explicitly we obtain

$$
\frac{\epsilon(z)}{\sigma^{2 / 3} N^{-1}}=F_{2}\left(\frac{z}{N \sigma^{1 / 3}}\right)
$$

where the scaling function $F_{2}(x)$ is given by

$$
\begin{aligned}
F_{2}(x) & =3 \alpha x\left(1-\alpha x^{2}\right)^{1 / 2} \\
\alpha & =\left(\frac{\pi^{2}}{12 \omega}\right)^{2 / 3}
\end{aligned}
$$

We show such a scaling plot for the chain-end density in Figure 3 together with the theoretical curve, eq 15, using $\omega=0.5$. Although some of the important features (such as the location and value of the peak) of the scaling function $F_{2}(x)$ obtained in the simulation are well reproduced by eq 15 , we note that there are some systematic deviations from the simulation data. In particular, MWC predict that $\epsilon(z)$ should tend to zero linearly with $z$, whereas we obtain a finite value for the chain-end density at $z=$ 0 . Also, the tail of the computed chain-end distribution does not show the abrupt decrease to zero predicted by MWC. It is difficult to determine whether these differences arise from the relatively small chains used in our simulations or from the fact that the theoretical calculations for $\epsilon(z)$ are based on a parabolic monomer density profile, which we have shown not to be accurate for small distances near the grafting plane. The quality of the scaling is better for $N=99$, where the graphs for the two largest coverages agree with each other, whereas the corresponding plots for $N=49$ show strong deviations from scaling. We attribute these deviations to the fact that we are not probably in the scaling regime for $N=49$.

B. Effect of Polydispersity. In another recent paper Milner, Witten, and Cates ${ }^{19}$ (hereafter to be referred as MWC2), extended the SCF formalism to study the effect of the polydispersity of the chain lengths on the monomer density profile. In particular, they calculated the 

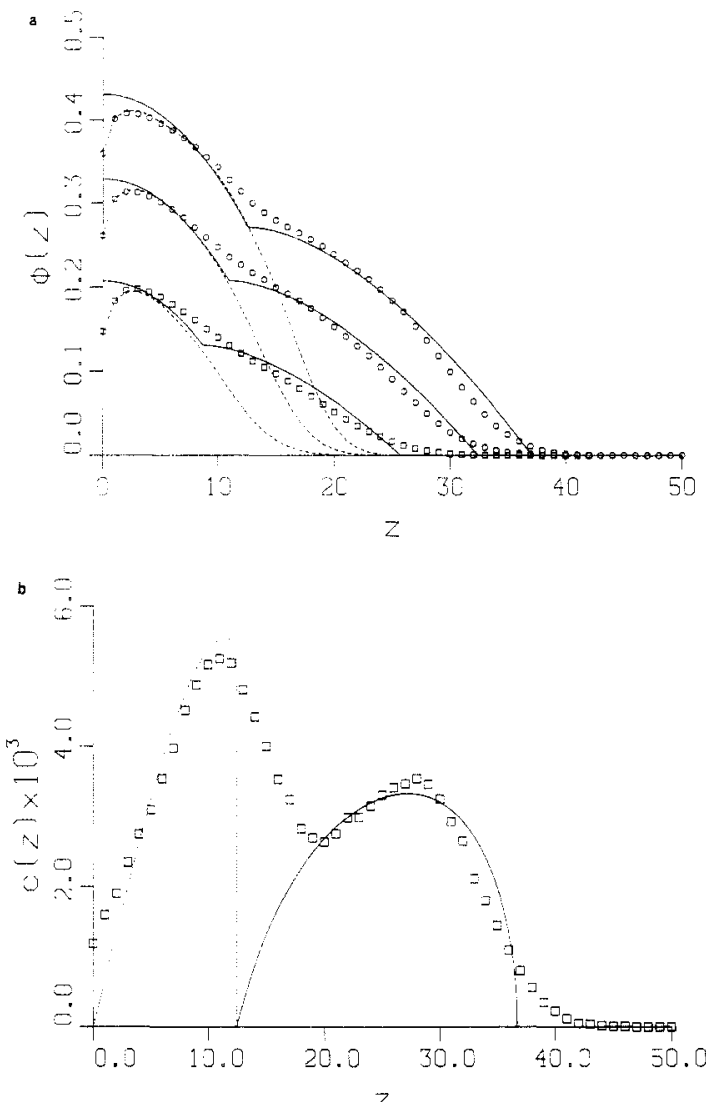

Figure 4. (a) The monomer density profile for a bimodal distribution function with chain lengths $N=49$ and $N^{\prime}=2 N=$ 98. The symbols $\square, 0$, and $\Delta$ correspond to $\sigma=0.04,0.08$, and 0.12 , respectively. The dotted lines correspond to data for density profiles for monodisperse chains with $N=49$. Note the perfect agreement between these two sets of results for distances close to the grafting surface. The solid lines are the corresponding MWC2 predictions. ${ }^{19}$ (b) The density of free chain ends for a bimodal distribution function with chain lengths $N$ $=49$ and $N^{\prime}=98$, and $\sigma=0.12$. The squares are the results of the simulations. The dotted line is the theoretical profile for $N=49$ monodisperse chains with $\sigma^{\prime}=\sigma / 2=0.06$ and for $z<$ $z_{1}$ (see text). The good agreement of the simulation data with this curve strongly suggest that the $N$ chains are mostly confined in this region. The solid line is the theoretical prediction for $z>z_{1}$ (eq 18b) with $\sigma=0.12$. Good agreement with this curve supports the MCW2 argument that all the long chain ends are at the outer end of the brush.

density profile for the cases of a bimodal and a uniform distribution of the chain lengths. In order to check the validity of these SCF calculations, we compute the monomer density profile for these two distributions for several values of surface coverage $\sigma$.

For the bimodal distribution, we consider two groups of chains, each group containing the same number of chains. The chain length in these two groups are chosen to be $N=49$ and $N^{\prime}=2 N=98$, respectively. In the case of $N^{\prime}=2 N$, MWC2 predict that the density profile should be that of a monodisperse distribution of chains with length $N$ up to a distance, $z_{1}$, given by

$$
z_{1}=h\left(1-2^{-2 / 3}\right)^{1 / 2}
$$

( $h$ is defined in eq 5 ), beyond which it should cross over to a functional form given by

$$
\varphi(z)=\left(A_{0}-\frac{B_{0} z_{1}^{2}}{4}\left(\frac{z}{z_{1}}+\frac{z_{1}}{z}\right)^{2}\right) \theta\left(h_{1}-z\right)
$$

where $A_{0}$ and $B_{0}$ are defined in eqs 6 and 7 and $h_{1}=h(1$ $\left.+2^{-2 / 3}\right)$. We show the density profiles in Figure $4 a$ for

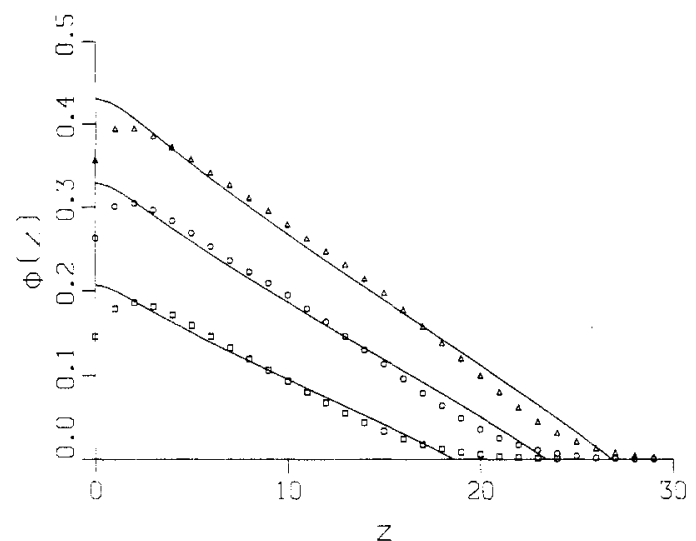

Figure 5. The monomer density profile for a uniform distribution function with chain lengths between $N_{1}=17$ and $N_{2}=$ 80. The symbols $\square, O$, and $\Delta$ correspond to $\sigma=0.04,0.08$, and 0.12 , respectively. The solid lines are the corresponding MWC2 predictions. ${ }^{19}$

several values of the surface coverage $\sigma$ along with the theoretical curves and the corresponding results for the monodisperse case with $N=49$. There is indeed a region close to the grafting surface where the profiles for the bimodal and monodisperse distributions agree with each other, even though the profiles cannot be approximated by a parabolic form as discussed earlier. Moreover, the two distributions start deviating at a distance $z_{1}{ }^{\prime}$ smaller than the predicted $z_{1}$. As the coverage increases, $z_{1}{ }^{\prime}$ approaches $z_{1}$ and the Monte Carlo profile develops hints of a singularity at this point. Of course, it is always difficult to observe a nonanalytic behavior in a numerical simulation because finite samples tend to smooth out any singularity.

We also calculated the chain-end density $\epsilon(z)$ for the bimodal distribution. Our results for $\sigma=0.12$ are shown in Figure 4b. The MWC2 theory predicts that all the $N$-chain free ends are found for $0<z<z_{1}\left(z_{1}\right.$ is defined in eq 16) and all the $N^{\prime}$-chain free ends are found beyond $z_{1}$. Explicitly, this theory predicts that

$$
\epsilon(z)=\frac{\pi^{2}}{4 \omega N^{3}} z\left(h^{2}-z^{2}\right)^{1 / 2} ; \quad 0<z<z_{1}
$$

and

$$
\epsilon(z)=\frac{(2)^{1 / 2}}{\pi \omega} B^{3 / 2} z_{1}{ }^{2}\left(h^{2}-y^{2}\right)\left(\frac{z}{z_{1}{ }^{2}}-\frac{z_{1}{ }^{2}}{z^{3}}\right) ; \quad z_{1}<z<h_{1}
$$

where $y=\left(z_{1} / 2\right)\left(\left(z / z_{1}\right)+\left(z_{1} / z\right)\right)$. In order to compare the simulation results with the MWC2 calculations, we show in Figure $4 \mathrm{~b}$ the corresponding end-density functions predicted in the theory. The agreement is reasonably good and thus supports the MWC2 argument of segregation by chain length (all ends of long chains being in the outer half of the brush).

For the uniform distribution case, we consider chain lengths uniformly distributed between $N_{1}=17$ and $N_{2}$ $=80$. The corresponding density profiles for three values of $\sigma$ are shown in Figure 5. By integrating MWC2 equations in this case, we obtain the functional form for the density profile $\varphi(z)$ (rather for the inverse function $z(\varphi))$ as

$$
\begin{aligned}
z(\varphi)=\frac{3\left(N_{2}-N_{1}\right)}{\pi} & \left(\frac{\omega A_{0}}{2}\right)^{1 / 2}\left[\left(\frac{4 N_{1}}{3\left(N_{2}-N_{1}\right)}+1-\frac{\varphi}{2 A_{0}}\right) \times\right. \\
& \left.\left(1-\frac{\varphi}{A_{0}}\right)^{1 / 2}-\frac{\varphi^{2}}{2 A_{0}^{2}} \cosh ^{-1}\left(\frac{A_{0}}{\varphi}\right)^{1 / 2}\right]
\end{aligned}
$$


The distribution is cutoff at $z=h_{2}=(1 / \pi)\left(\omega A_{0} / 2\right)^{1 / 2} \times$ $\left(3 N_{2}+N_{1}\right)$. We show the corresponding curves in Figure 5 as well. Apart from the depletion zone near the surface, the agreement between Monte Carlo results and the analytical theory is remarkable.

\section{Conclusions}

We have carried out a detailed Monte Carlo simulation for the grafted polymer brush for several values of surface coverages and computed the monomer density profile for both monodisperse and polydisperse distributions of chain lengths. The simulations are computationally very extensive since we consider a large number of long chains in the system in the presence of excludedvolume interactions. Also special care is needed in letting the system properly relax to equilibrium before any measurements are carried out. If each chain in the system is not equilibrated over a time much larger than that of a single isolated chin, we observed some unphysical results. This is particularly true for large values of surface coverages.

For the monodisperse case, the monomer density profile computed in our simulations shows a depletion zone near the grafting plane in agreement with phenomenological arguments. ${ }^{7}$ Beyond this depletion layer, the density profile can be approximated by a parabolic form. This result is consistent with recent analytical calculation $\mathrm{s}^{16}$ but not with the scaling arguments, which sug. gest a plateau region for the density profile. Moreover the free chain ends are not excluded from near the grafting plane in agreement with ref 16 . We point out that in the comparison of the theory and the Monte Carlo simulations, only one parameter needs to be determined, namely, the strength of the excluded-volume interactions, $\omega$.

In the case of a system consisting of two species of polymers of length $\mathrm{N}$ and $2 N$, we find that there is a region in which the density profile matches that of the monodisperse case with chain length $N$, in agreement with ref 19. The width of this region, however, is narrower than predicted by the theory, and no parabolic fit seems to be valid for distances near the grafting plane. The density of free chain ends agree reasonably well with MWC2 predictions, and we find that almost all the ends of longer chains are in the outer region of the brush.

We have also considered a uniform distribution of chain lengths and compared the density profile with the functional form obtained by integrating the equations derived in the SCF formalism of Milner, Witten, and Cates. The agreement between the Monte Carlo data and the theory is remarkable except, again, for the presence of a depletion layer near the grafting plane.

Finally, we note that it will be interesting the calculate the force profiles by squeezing the brush against a wall at the front, since this kind of calculation is quite relevant for experimental situations. ${ }^{11}$ For this purpose one needs to calculate the free energy of the system which is difficult to calculate in Monte Carlo calculations. How- ever, we point out that methods for determining the pressure in lattice simulations have been developed ${ }^{24}$ in recent years. These methods can be generalized to the case of grafted chains as well and will be addressed in future publications. ${ }^{25}$

Acknowledgment. We thank M. S. El-Aasser, J. D. Gunton, D. C. Hong, and M. Muthukumar for valuable discussions. We also thank E. T. Gawlinski and the Center for Advanced Computational Science in Temple University for a generous amount of computer time in their FPS-264 computers. A.C. is supported by NSF Grant DMR-8612609. R.T. acknowledges financial support from DGICYT Project No. PB-86-0534 (Spain).

\section{References and Notes}

(1) Napper, D. H. Polymeric Stabilization of Colloidal Dispersions; Academic Press: London, 1983.

(2) Tadros, Th. F. The Effects of Polymers on Dispersion Properties; Academic Press: London, 1982.

(3) Takahashi, A.; Kawaguchi, M. Adv. Polym. Sci. 1982, 46, 1.

(4) Vincent, B.; Whittington, S. Surf. Colloid Sci. 1981, 12, 1.

(5) Fleer, G. J.; Lykema, J. In Adsorption from Solution at the Solid/Liquid Interface; Academic Press: London, 1983.

(6) Alexander, S. J. Phys. (Les Ulis, Fr.) 1977, 38, 983.

(7) See, for example: de Gennes, P.-G. Adv. Colloid Interface Sci. $1987,27,189$ for a review of scaling arguments. Also see: de Gennes, P.-G. J. Phys. (Les Ulis, Fr.) 1976, 37, 1443. de Gennes, P.-G. Macromolecules 1980, 13, 1069.

(8) Dolan, A. K.; Edwards, S. F. Proc. R. Soc. London 1974, 337, $509 ; 1975,343,427$.

(9) Scheutjens, J.; Fleer, G. J. J. Chem. Phys. 1979, 83, 1619; Macromolecules $1985,18,1882$.

(10) Ben-Shaul, A.; Szleifer, I.; Gelbart, W. M. J. Chem. Phys. 1985, 83, 3597. Viouvy, J. L.; Gelbart, W. M.; Ben-Shaul, A. J. Chem. Phys. 1987, 87, 4114 .

(11) For a review of experimental results, see: Cohen-Stuart, M. A.; Cosgrove, T.; Vincent, B. Adv. Colloid Interface Sci. 1986, 24, 143. See also: Taunton, H. J.; Toprakcioglu, C.; Fetters, L. J.; Klein, J. Nature 1988, 332, 712. Hadziioannou, G.; Patel, S.; Granick, S.; Tirrell, M. J. Am. Chem. Soc. 1986, 108, 2869.

(12) Gerber, P. R.; Moore, M. A. Macromolecules 1977, $20,476$. Semenov, A. N. Sov. Phys.-JETP (Engl. Transl.) 1985, 61, 733.

(13) Muthukumar, M.; Ho, J. S. Macromolecules 1989, 22, 965.

(14) Hirz, S. M.S. Thesis, University of Minnesota, 1986, unpublished work.

(15) Cosgrove, T.; Heath, T.; van Lent, B.; Leermakers, F.; Scheutjens, J. Macromolecules 1987, 20, 1692.

(16) Milner, S. T.; Witten, T. A.; Cates, M. E. Macromolecules $1988,21,2610$

(17) Clark, A. T.; Lal, M. J. Chem. Soc., Faraday Trans. 2 1975, 74,1857 .

(18) Murat, M.; Grest, G. S. Macromolecules 1989, 22, 4054.

(19) Milner, S. T.; Witten, T. A.; Cates, M. E. Macromolecules $1989,22,853$.

(20) Kolinski, A.; Skolnick, J.; Yaris, R. J. Chem. Phys. 1986, 84, 1922.

(21) Verdier, P. H.; Stockmayer, W. H. J. Chem. Phys. 1962, 36, 227. Domb, C. Adv. Chem. Phys. 1962, 15, 229. Hilhorst, H. J.; Deutch, J. M. J. Chem. Phys. 1975, 63, 5153.

(22) Gurler, M. T.; Crabb, C. C.; Dahlin, D. M.; Kovac, J. Macromolecules 1983, 16, 398.

(23) There is a misprint in eq 18 of ref 16 . That equation for the chain-end density $\epsilon(z)$ is not properly normalized.

(24) Dickman, R. J. Chem. Phys. 1987, 87, 2246 and references therein.

(25) Chakrabarti, A.; Toral, R. Unpublished results. 